\title{
Editorial
}

\section{Nanostructured Materials for Lithium-Ion Batteries}

\author{
Jianmin Ma, ${ }^{1}$ Yongzhu Fu, ${ }^{2}$ Bo Yu, ${ }^{3}$ and Jun $\mathrm{Zhang}^{4}$ \\ ${ }^{1}$ Key Laboratory for Micro-Nano Optoelectronic Devices of Ministry of Education, College of Physics and Microelectronics, \\ Hunan University, Changsha 410082, China \\ ${ }^{2}$ Materials Science and Engineering, The University of Texas at Austin, Austin, TX 78712, USA \\ ${ }^{3}$ Functional Nanomaterials Laboratory, Solar and Photovoltaics Engineering Research Center, \\ King Abdullah University of Science and Technology (KAUST), Thuwal 23955-6900, Saudi Arabia \\ ${ }^{4}$ School of Materials Science and Engineering, University of Jinan, Jinan 250022, China \\ Correspondence should be addressed to Jianmin Ma; nanoelechem@hnu.edu.cn
}

Received 13 May 2013; Accepted 13 May 2013

Copyright ( 2013 Jianmin Ma et al. This is an open access article distributed under the Creative Commons Attribution License, which permits unrestricted use, distribution, and reproduction in any medium, provided the original work is properly cited.

In the past decade, lithium-ion (Li-ion) batteries have been considered as one of the viable alternative technologies for applications such as electrical vehicles and grid energy storage for renewable energies (e.g., solar and wind) due to their high energy density and long cycle life. Recent nanotechnology has acted as the positive catalysts for the development of advanced electrode materials for high-performance Li-ion batteries. In this issue, some typical electrode nanomaterials have been reviewed and discussed.

The paper "Graphene-based composites as cathode materials for lithium ion batteries" by L. Chen et al. reviews the recent advances in graphene-based composites and their application as cathode materials for Li-ion batteries. They focus on the synthesis methods of graphene-based composites and their superior electrochemical performance in Li-ion batteries.

In the paper "A review on the synthesis of manganese oxide nanomaterials and their applications on lithium-ion batteries" by $\mathrm{X}$. Liu et al., the authors briefly review the recent development of manganese oxide nanomaterials. They present two important viewpoints: (i) simple and effective methods should be explored for the synthesis of manganese oxide nanomaterials with high surface areas and good dispersity in the future; (ii) the electrochemical mechanism of manganese oxide nanomaterials should be deeply understood.

The paper " $3 D$ self-supported nanoarchitectured arrays electrodes for lithium-ion batteries" by X. Chen reviews recent advances in the strategies for the fabrication, selection of the different current collector substrates, and structural configuration of three-dimensional self-supported nanoarchitectured arrays electrodes with different cathode and anode materials. It also discussed the intrinsic relationship of the unique structural characters, the conductive substrates, and electrochemical kinetic properties of three-dimensional selfsupported nanoarchitectured arrays electrodes. In addition, It highlights the future design trends and directions of three-dimensional self-supported nanoarchitectured arrays electrodes for future advanced Li-ion batteries.

In conclusion, Li-ion batteries, as one of the most promising electrochemical energy storage technologies, will play a significant role in many applications. To further improve the energy density and cycle life, electrodes based on nanotechnologies (e.g., nanostructure, nanofabrication, and nanoframework) are believed to be the most promising strategy. To achieve these, new methods to synthesize nanomaterials need to be explored, in-depth understanding of the electrochemical phenomena in nanoscale needs to be enhanced, and new tools to characterize nanomaterials need to be developed. With the successful development of high energy density, long cycle life Li-ion batteries, our society will become efficient and sustainable.

Jianmin $\mathrm{Ma}$ Yongzhu Fu Bo $\mathrm{Yu}$ Jun Zhang 

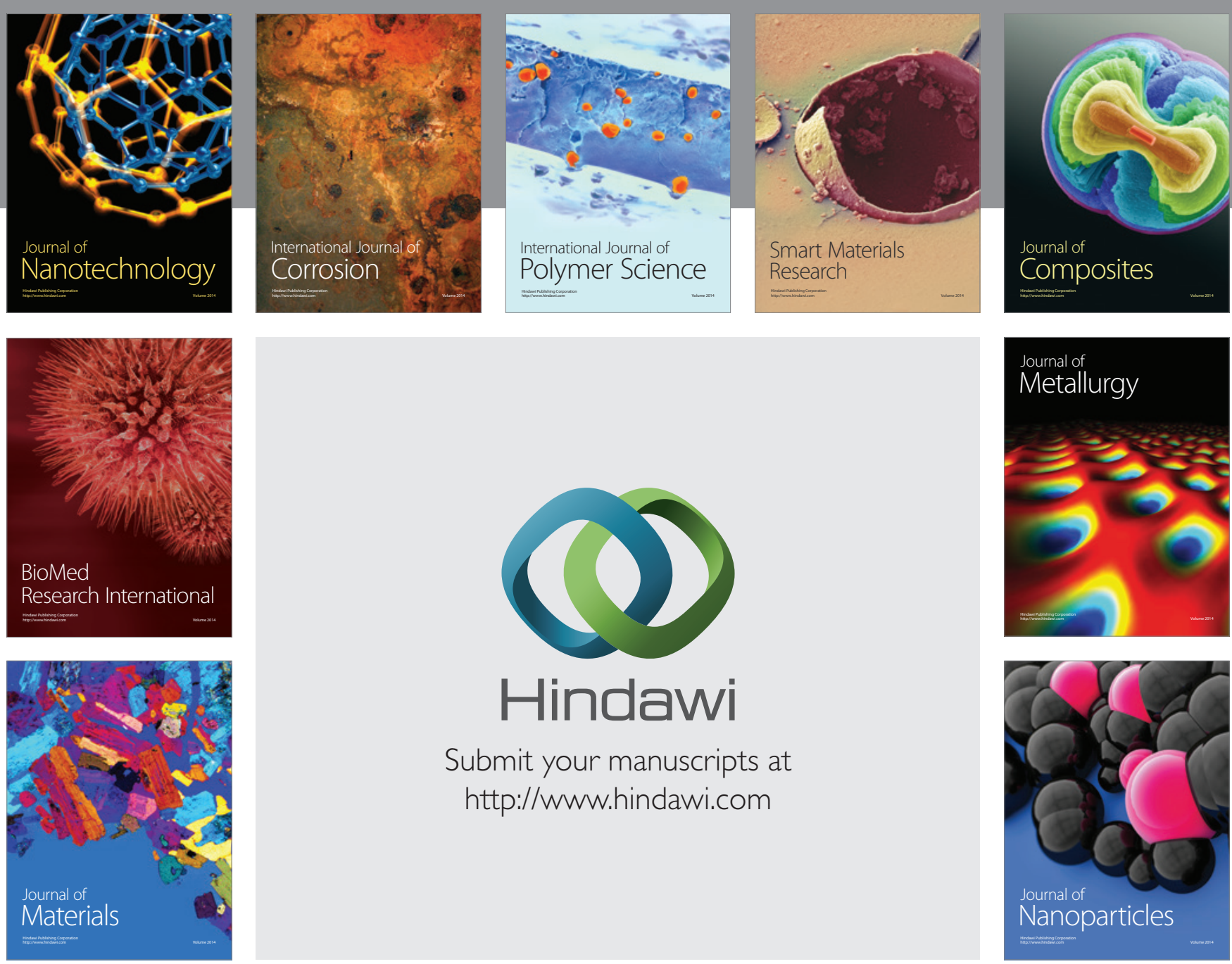

Submit your manuscripts at http://www.hindawi.com
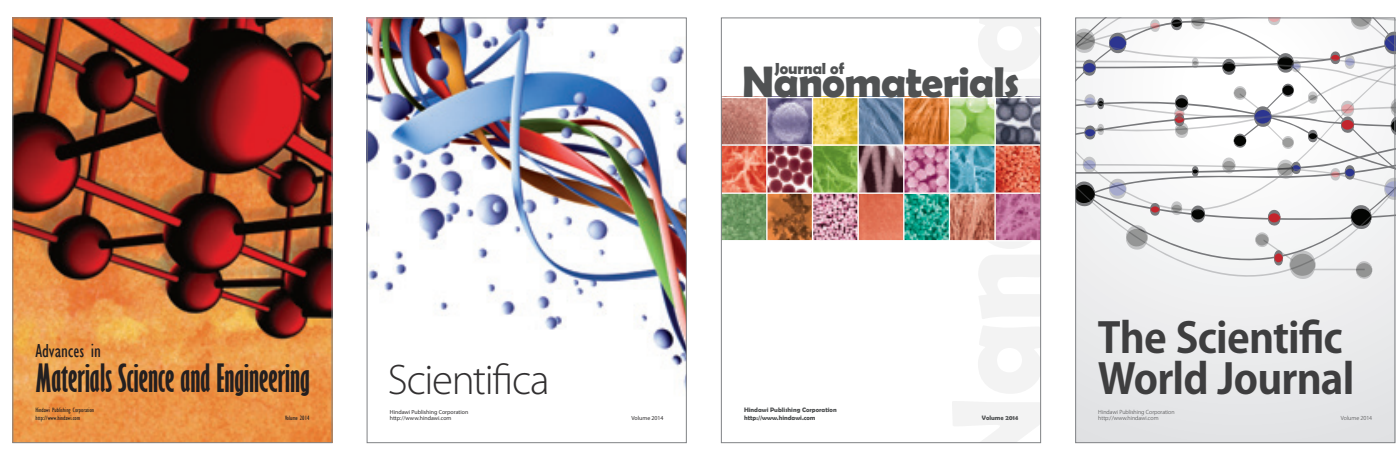

\section{The Scientific World Journal}
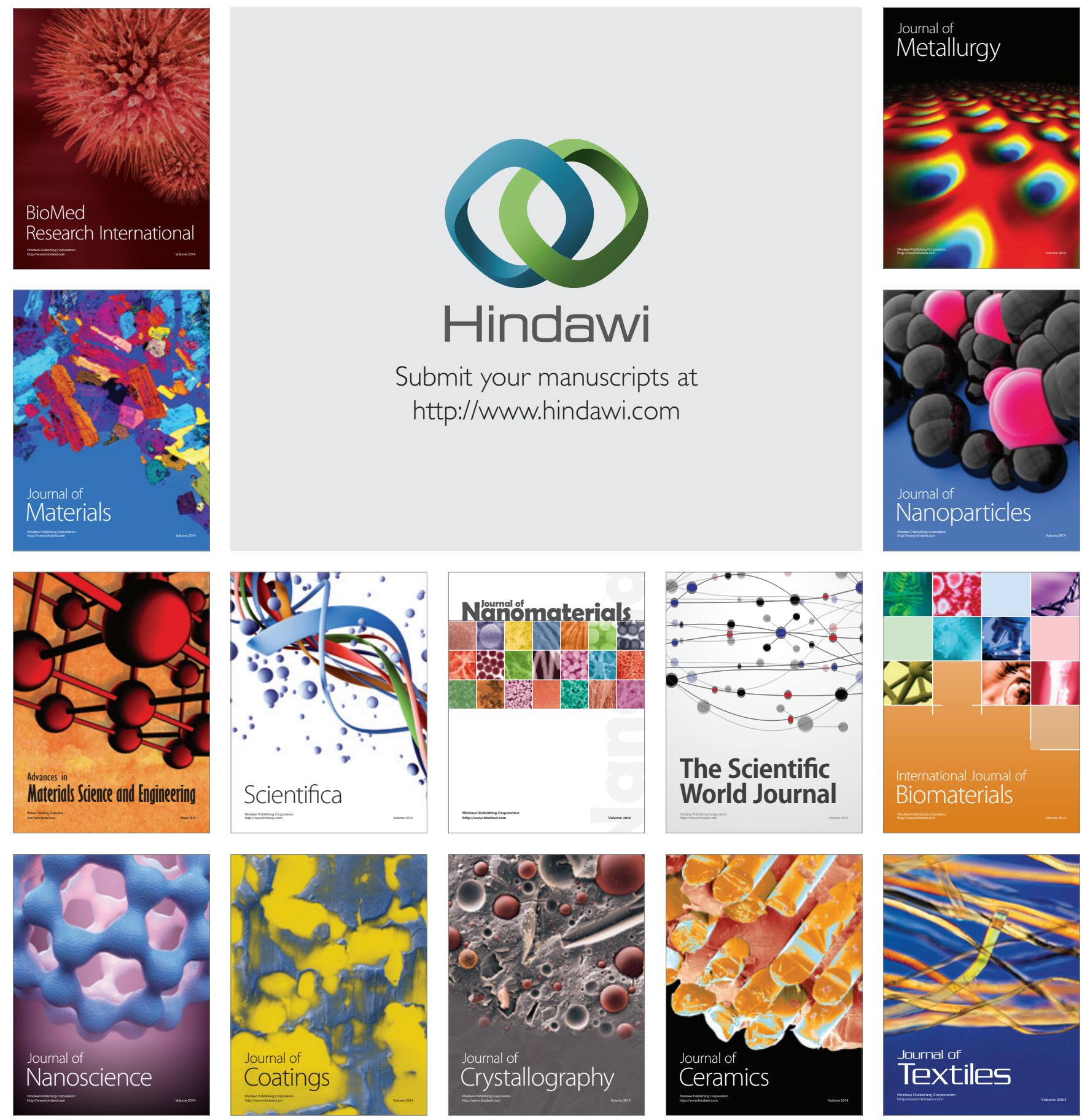